\title{
Effect of Diabetes Mellitus and Metformin Usage on Treatment Outcomes and Side Effects on Prostate Cancer Treated with Radical Radiotherapy
}

\author{
๑ Meltem Dağdelen, ๑ Ceren Barlas, ๑ Cumhur Yıldırım, ๑ Songül Çavdar Karaçam, ๑ H. Fazilet Öner Dinçbaş \\ Istanbul University-Cerrahpaşa, Cerrahpaşa Faculty of Medicine, Department of Radiation Oncology, Istanbul, Turkey
}

\begin{abstract}
Objective: Diabetes mellitus (DM) is a common comorbidity in patients with prostate cancer. Radiotherapy was reported to induce acute and late side effects in patients with DM due to vascular damage. Moreover, some studies have shown that metformin, an oral antidiabetic drug, can reduce biochemical and disease recurrence in patients with prostate cancer. This study aimed to evaluate retrospectively the effect of metformin on biochemical disease control and to observe the acute and late side effects of prostate cancer treated with radiotherapy.

Materials and Methods: This study enrolled 94 patients who received radical radiotherapy between 2010 and 2017 . However, out of 22 patients with DM, 17 received metformin and five received metformin plus insulin treatment. Biochemical recurrence-free survival (bRFS), overall survival, and side effects were assessed between patients with and without DM.

Results: The median follow-up time was 57 (15-128) months. The 5-year bRFS rate in patients with and without DM were 100\% and 89.2\%, respectively ( $p=0.10)$. Acute grade 1-2 side effects were observed in all patients with DM, while $56(78 \%)$ patients without DM had acute side effects, and the difference is significant $(p=0.02)$. Acute grade 3 genitourinary and gastrointestinal toxicity was found in one patient without $D M$, whereas late grade 3 gastrointestinal toxicity was observed in one patient with DM.

Conclusion: Although patients with DM were found to have better bRFS than patients without DM, we could not show the benefit of metformin, and the difference was not significant. By contrast, acute side effects were significantly higher in patients with DM.
\end{abstract}

Keywords: Prostate cancer, diabetes mellitus, metformin, radiotherapy, side effects

\section{Introduction}

Prostate cancer is the most common cancer in men, the second leading cause of cancer-related death, and usually observed in older men (1). Diabetes mellitus (DM) is a common chronic endocrine disease developed by either genetically or acquired deficiency. Type 1 and type 2 are the common forms of DM, and more than $90 \%$ of patients have type 2 DM (2). Type 2 DM is mainly caused by insulin resistance, particularly common in the older population, and the prevalence of DM in individuals aged $>65$ years is $26.9 \%$ (3). Therefore, coexisting diagnoses of prostate cancer and DM increased because of aging. Metformin is an orally administered and frequently used as a insulin sensitizer drug that belongs to the biguanide antidiabetic family. Recently, the antineoplastic activity of this compound shown in some in vitro models is gaining interest (4). Several retrospective studies have demonstrated that metformin treatment can reduce the incidence of prostate cancer, prostate-specific antigen (PSA) levels, and disease recurrence $(5,6)$.

Especially, breast, colorectal, endometrium, liver, and pancreatic cancers occur more commonly in individuals with DM, and the prevalence of DM in patients newly diagnosed with cancer is even higher, ranging from $8 \%$ to $18 \%$ (7). DM can cause longterm complications, such as cardiovascular disease, retinopathy, and neuropathy. Patients with both cancer and DM have an increased risk of long-term mortality in comparison with patients without DM (8).

Radiotherapy is one of the main treatment modalities for locally advanced prostate cancer. Management and side effects of prostate cancer treatment are particularly affected by comorbidities. Some studies have reported that patients with DM experienced more radiation-induced genitourinary and gastrointestinal system side effects than patients without DM after prostate cancer radiotherapy (9).

Cite this article as: Dağdelen M, Barlas C, Yıldııım C, Çavdar Karaçam S, Öner Dinçbaş HF. Effect of Diabetes Mellitus and Metformin Usage on Treatment Outcomes and Side Effects on Prostate Cancer Treated with Radical Radiotherapy. Bull Urooncol 2021;20(3):162-167

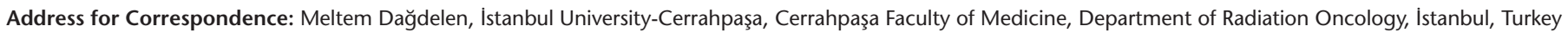
Phone: +90 5426603810 E-mail: meltemdagdelen@windowslive.com ORCID-ID: orcid.org/0000-0002-2009-0002 Received: 20.07.2020 Accepted: 17.12.2020 
This single-center retrospective study aimed to evaluate the effect of metformin on biochemical disease control and to observe the acute and late side effects of prostate cancer treated with definitive radiotherapy.

\section{Materials and Methods}

\section{Study Population}

This study included 94 consecutive patients with prostate cancer treated by definitive radiotherapy between 2010 and 2017 . Moreover, 22 (23\%) patients had DM and received metformin treatment $(1.000 \mathrm{mg} /$ day), while five patients received insulin treatment in addition to metformin treatment. Metformin therapy had varied duration. Patients had T1-T2 (79\%) and T3T4 (21\%) disease. At presentation, 16 (17\%) patients had highgrade tumors (Gleason score $8 \leq$ ) and 43 (46\%) had high-risk disease. The cohort comprised of $26 \%$ low, $28 \%$ medium, and $46 \%$ high-risk groups according to the National Comprehensive Cancer Network risk category (10). Luteinizing hormonereleasing hormone agonists were used for 6 months in 16 and for 24 months in 43 patients as androgen deprivation therapy. Characteristics of the patients are listed in Table 1.

\section{Treatment Preparation and Planning}

Patient preparation was performed before radiotherapy planning computer tomography (CT) and every treatment fraction as reported previously (11). The patients were asked to avoid eating gas-producing food and to consume a low-fiber diet before simulation and during treatment. Organs at risk and target volumes were contoured according to Radiation Therapy Oncology Group guidelines in planning CT.

\begin{tabular}{|l|l|}
\hline Table 1. Patient characteristics & n (\%) \\
\hline Patient characteristics & $4(4 \%)$ \\
\hline T-stage & $70(75 \%)$ \\
\hline T1 & $17(18 \%)$ \\
\hline T2 & $3(3 \%)$ \\
\hline T3 & \multicolumn{2}{|l|}{} \\
\hline T4 & $5(5 \%)$ \\
\hline N-stage & $24(26 \%)$ \\
\hline N1 & $27(28 \%)$ \\
\hline Risk groups & $43(46 \%)$ \\
\hline Low & \multicolumn{2}{|l}{} \\
\hline Intermediate & $16(17 \%)$ \\
\hline High & $43(46 \%)$ \\
\hline Androgen deprivation therapy & $22(23 \%)$ \\
\hline Short-term & $17(18 \%)$ \\
\hline Long-term & $5(5 \%)$ \\
\hline Diabetic patients & \multicolumn{2}{|l}{} \\
\hline DM treatments & \\
\hline Metformin & \\
\hline Metformin + insulin & \\
\hline DM: Diabetes mellitus & \\
\hline
\end{tabular}

\section{Treatment}

Intensity-modulated radiotherapy plans were generated for each patient using the Eclipse version 8.6 treatment planning system by using $6 \mathrm{MV}$ photon beams. The median dose of radiation therapy was 78 Gy (range, 70-80 Gy) in 39 (range, 2840) fractions. Only prostate volume is irradiated in 78 patients, whereas pelvic lymphatics were added to the treatment volumes in 16 patients. Field verification for image-guided radiation therapy was carried out with cone-beam CT every day.

\section{Follow-up}

During radiotherapy, all patients were examined once a week for urinary symptoms such as dysuria, urinary incontinence, and hematuria, gastrointestinal symptoms such as the number of daily defecation and stool density, and complaints about abdominal pain and gas. After the radiotherapy, patients were followed up every 3 months for the first 2 years, every 6 months between 2 and 5 years, and annually after 5 years. PSA was evaluated at each follow-up, and additional examinations were postulated according to the PSA result. All patients were examined at each visit and assessed for late toxicity.

\section{Statistical Analysis}

This study was conducted retrospectively. Biochemical recurrence-free survival (bRFS) was defined as the time from the end of radiotherapy to PSA recurrence. Kaplan-Meier survival analysis was performed for medicine use (i.e., antidiabetic drugs) with the endpoint of bRFS. Univariate and multivariate analyses performed by Cox-regression method were adjusted for the baseline characteristics, including age, stage, Gleason score, PSA, treatment field, radiotherapy doses, androgen deprivation therapy, doses of organs at risk, other comorbidities (hypertension and coronary artery disease), and DM. Common Terminology Criteria for Adverse Events v5.0 was used for the evaluation of acute and late gastrointestinal and genitourinary side effects. Comparisons of acute and chronic side effects for patients with and without DM were made by the chi-square test. The retrospective study protocol was approved by the Ethics Committee of Istanbul University-Cerrahpasa, Cerrahpasa Faculty of Medicine and the study was conducted in accordance with the tenets of the Helsinki Declaration. SPSS version 21 for Windows (IBM Corp., Armonk, NY) was used for all statistical analyses, and $p<0.05$ was considered for significance.

\section{Results}

The median follow-up time was 57 (15-128) months, and the median age was 69 (53-88) years. Thirteen patients died; however, only three of them died from prostate cancer. PSA relapse was observed in eight patients without DM. The 5-year and 8-year overall survival (OS) for the total study population were $91.4 \%$ and $75.4 \%$, respectively (Figure 1 ). Patients aged $\geq 70$ years were significantly associated with a higher risk of mortality $[p=0.023$, confidence interval $(\mathrm{Cl})=0.22(0.06-0.81)]$ than patients aged $<70$ years in the univariate analyses. Results of the univariate and multivariate analyses are listed in Table 2.

In this study, the 5-year and 8-year bRFS rates were $91.6 \%$ and $89.5 \%$, respectively. In the multivariate analyses, Gleason score 


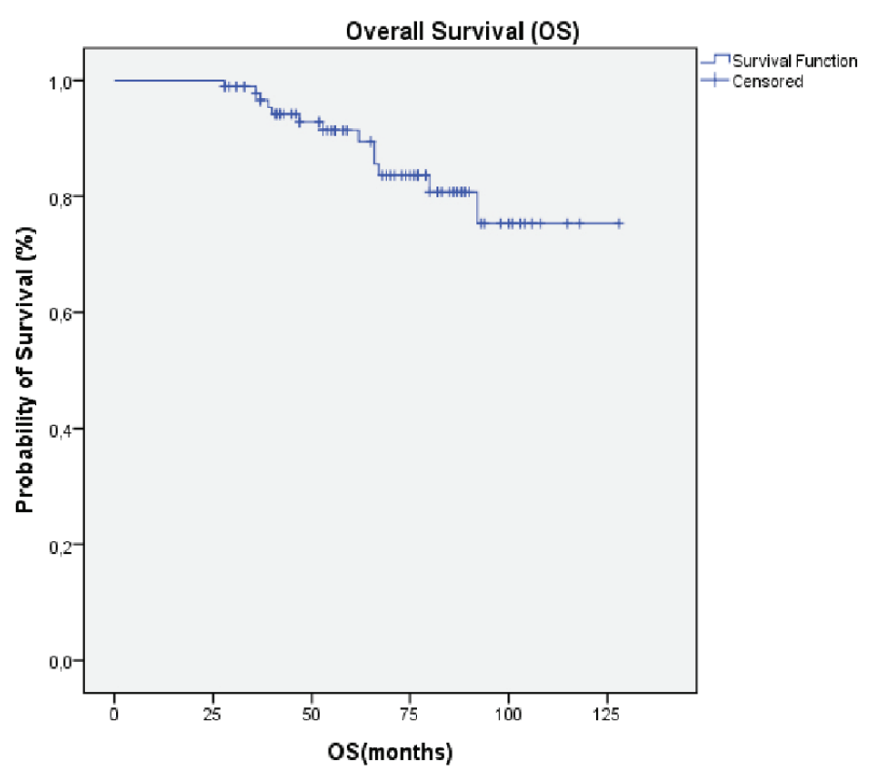

Figure 1. Overall survival for patients

\begin{tabular}{|c|c|c|c|c|}
\hline Characteristic & $\begin{array}{l}\text { Univariate HR } \\
(95 \% \mathrm{Cl})\end{array}$ & p-value & $\begin{array}{l}\text { Multivariate } \\
\text { HR }(95 \% \mathrm{Cl})\end{array}$ & p-value \\
\hline \multicolumn{3}{|l|}{ Risk category } & \multirow{3}{*}{ NS } & \\
\hline Intermediate & $0.78(0.17-3.54)$ & 0.73 & & \\
\hline High & $0.70(0.19-2.53)$ & 0.59 & & \\
\hline Older age & $4.53(1.24-16.59)$ & 0.02 & $\begin{array}{l}4.87 \\
(1.30-18.24) \\
\end{array}$ & 0.019 \\
\hline PSA level & $0.99(0.33-2.99)$ & 0.94 & NS & \\
\hline TNM stage & $1.03(0.28-3.76)$ & 0.96 & NS & \\
\hline \multicolumn{3}{|l|}{ ADT } & \multirow{3}{*}{ NS } & \\
\hline Short-term & $2.49(0.55-11.18)$ & 0.23 & & \\
\hline Long-term & $1.22(0.30-4.93)$ & 0.78 & & \\
\hline Pelvic nodal RT & $1.42(0.31-6.55)$ & 0.65 & NS & \\
\hline DM status & $2.6(0.33-20.1)$ & 0.30 & $\begin{array}{l}1.36 \\
(0.16-11.29)\end{array}$ & 0.775 \\
\hline Recurrence & $4.64(1.43-15.11)$ & 0.01 & $\begin{array}{l}5.12 \\
(1.51-17.34)\end{array}$ & 0.009 \\
\hline
\end{tabular}

$\geq 8(\mathrm{p}=0.003 ; \mathrm{Cl}=0.11(0.03-0.49)$ and age $<60$ years $(\mathrm{p}=0.019 ;$ $\mathrm{Cl}=0.19(0.05-0.76)$ were found to be negative factors for bRFS.

Subgroup analyses showed similar OS and bRFS rates. The 5 -year OS rates in patients with and without DM were 93\% and $91 \%$, respectively $(p=0.30)$ (Figure 2$)$. The 5 -year bRFS rates in patients with and without DM were $100 \%$ and $89.2 \%$, respectively $(p=0.10)$ (Figure 3$)$. A comparison of the survival results of patients with and without DM are listed in Table 3.

As regards side effects, acute grade 1-2 side effects were observed in all patients with DM, whereas $78 \%$ of patients without DM had acute side effects, and the difference is significant $(p=0.02)$

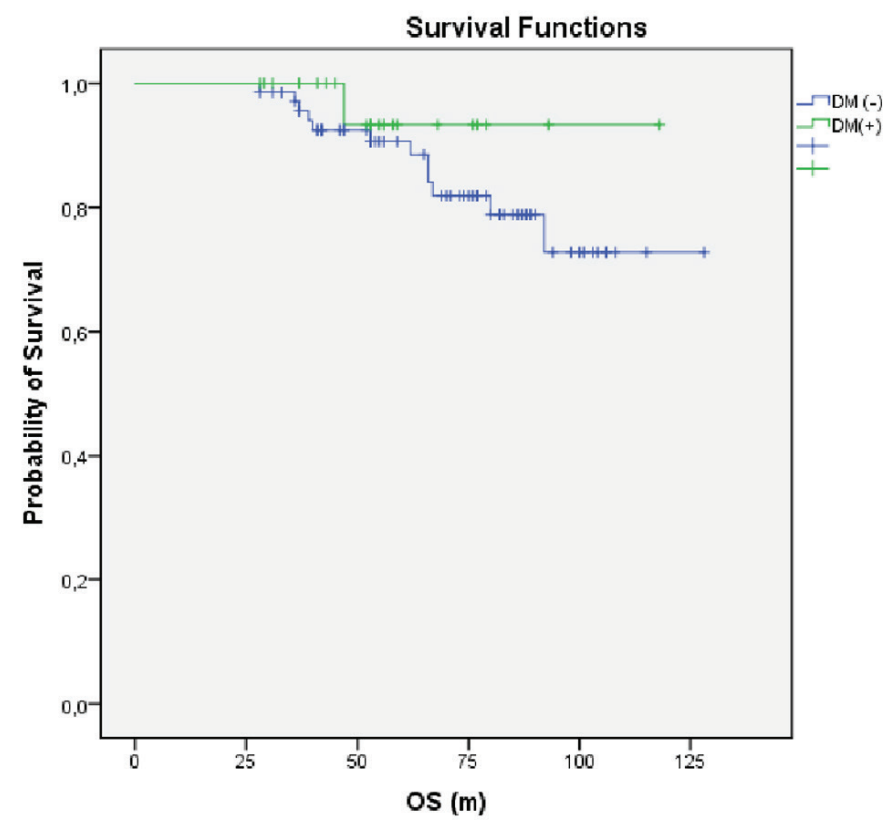

Figure 2. Overall survival for diabetic and non-diabetic patients

\begin{tabular}{|c|c|c|c|c|c|}
\hline & \multicolumn{2}{|c|}{ DM (-) } & \multicolumn{2}{|c|}{$\mathrm{DM}(+)$} & \multirow[t]{2}{*}{$p$} \\
\hline & $\mathbf{n}$ & $\%$ & $\mathbf{n}$ & $\%$ & \\
\hline \multicolumn{6}{|c|}{ Risk category } \\
\hline Low & 18 & 25 & 6 & 27.3 & \multirow{3}{*}{0.557} \\
\hline Medium & 19 & 26.4 & 8 & 36.4 & \\
\hline High & 35 & 48.6 & 8 & 36.4 & \\
\hline \multicolumn{5}{|l|}{ PSA } & \multirow{3}{*}{0.199} \\
\hline$<10$ & 33 & 45.8 & 13 & 59.1 & \\
\hline$\geq 10$ & 39 & 54.2 & 9 & 40.9 & \\
\hline \multicolumn{5}{|l|}{ TNM } & \multirow{3}{*}{0.138} \\
\hline Stage 1-2 & 53 & 73.6 & 19 & 86.4 & \\
\hline Stage 3-4 & 19 & 26.4 & 3 & 13.6 & \\
\hline \multicolumn{5}{|l|}{ Age } & \multirow{3}{*}{0.313} \\
\hline$<70$ & 37 & 51.4 & 14 & 63.6 & \\
\hline$\geq 70$ & 35 & 48.6 & 8 & 36.4 & \\
\hline \multicolumn{5}{|l|}{ ADT } & \multirow{4}{*}{0.213} \\
\hline None & 26 & 36.1 & 9 & 40.9 & \\
\hline Short-term & 10 & 13.9 & 6 & 27.3 & \\
\hline Long-term & 36 & 50 & 7 & 31.8 & \\
\hline \multicolumn{5}{|c|}{ Pelvic nodal RT } & \multirow{3}{*}{0.315} \\
\hline Absent & 59 & 81.9 & 20 & 90.9 & \\
\hline Present & 13 & 18.1 & 2 & 9.1 & \\
\hline
\end{tabular}

(Table 4). Urinary side effects were more common in all patients. Side effects such as dysuria, nocturia, urinary incontinence, pollakiuria, and hematuria were observed in 95.5\%, 45.5\%, $22.5 \%, 18.1 \%$, and $4.5 \%$ of the patients, respectively. Six 
patients experienced diarrhea as an acute gastrointestinal side effect. Late side effects especially dysuria and nocturia were found in $23 \%$ and $13 \%$ of the patients, respectively $(p=0.26)$ (Table 5). Acute grade 3 genitourinary and gastrointestinal toxicity was observed in one patient without DM, whereas late grade 3 gastrointestinal toxicity was seen in one patient with DM.

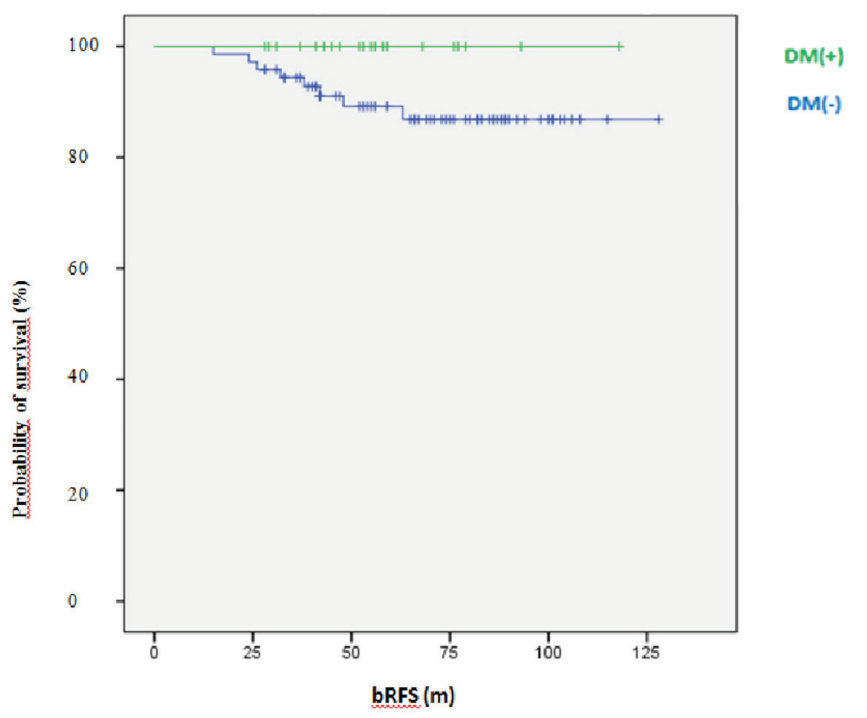

Figure 3. Biochemical recurrence free survival (bRFS) for diabetic and nondiabetic patients

\begin{tabular}{|c|c|c|c|c|c|}
\hline \multirow[t]{2}{*}{ DM } & \multicolumn{4}{|c|}{ Acute gastrointestinal side effects } & \multirow[t]{2}{*}{ Total } \\
\hline & Absent & Grade 1 & Grade 2 & Grade 3 & \\
\hline Diabetic & $16(73 \%)$ & $4(18 \%)$ & $2(9 \%)$ & $0(0 \%)$ & $\begin{array}{l}22 \\
(100 \%)\end{array}$ \\
\hline \multirow[t]{3}{*}{ Non-diabetic } & $54(75 \%)$ & $9(12 \%)$ & $7(10 \%)$ & $2(3 \%)$ & $\begin{array}{l}72 \\
(100 \%)\end{array}$ \\
\hline & \multicolumn{4}{|c|}{ Acute genitourinary side effects } & \\
\hline & Absent & Grade 1 & Grade 2 & Grade 3 & \\
\hline Diabetic & $2(9 \%)$ & $\begin{array}{l}18 \\
(82 \%)\end{array}$ & $2(9 \%)$ & $0(0 \%)$ & $\begin{array}{l}22 \\
(100 \%)\end{array}$ \\
\hline Non-diabetic & $\begin{array}{l}18 \\
(25 \%)\end{array}$ & $\begin{array}{l}34 \\
(47 \%)\end{array}$ & $19(26 \%)$ & $1(2 \%)$ & $\begin{array}{l}72 \\
(100 \%)\end{array}$ \\
\hline
\end{tabular}

Table 5. Late side-effects of patients

\begin{tabular}{|l|l|l|l|l|l|}
\hline DM & \multicolumn{4}{|l|}{ Late gastrointestinal side effects } & Total \\
\hline & Absent & Grade 1 & Grade 2 & Grade 3 & \\
\hline Diabetic & $19(85 \%)$ & $1(5 \%)$ & $1(5 \%)$ & $1(5 \%)$ & $22(100 \%)$ \\
\hline Non-diabetic & $69(96 \%)$ & $1(1 \%)$ & $2(3 \%)$ & $0(0 \%)$ & $72(100 \%)$ \\
\hline & Late genitourinary side effects & \\
\hline & Absent & Grade 1 & Grade 2 & Grade 3 & \\
\hline Diabetic & $18(82 \%)$ & $3(14 \%)$ & $1(4 \%)$ & $0(0 \%)$ & $22(100 \%)$ \\
\hline Non-diabetic & $63(88 \%)$ & $8(10 \%)$ & $1(2 \%)$ & $0(0 \%)$ & $72(100 \%)$ \\
\hline DM: Diabetes mellitus & & & \\
\hline
\end{tabular}

\section{Discussion}

Prostate cancer is the most common male cancer and the second leading cause of death among other malignancies. The incidence rates of prostate cancer and DM are increasing in the last decades. At present, treatment guidelines recommend metformin as the first-line therapy for DM (12). Metformin is an insulin sensitizer and a potent adenosine monophosphateactivated protein kinase activator. It inhibits the mammalian target of rapamycin complex-1 pathway in carcinogenesis (13). In the last decades, many studies have investigated the effect of DM and metformin on cancer incidence and mortality (11). It is believed that metformin may have a greater effect on cancer survival by modulating cellular energy rather than the transformation of benign cells to malignant cells.

Studies examining the influence of metformin on prostate cancer have inconsistent results. In addition to cancer incidence, several studies have investigated the role of metformin on prostate cancer-specific mortality as well as recurrence. However, data are limited about the positive effect of metformin on treatment results with radiotherapy. Spratt et al. (8) conducted a retrospective study and revealed that metformin may improve bRFS, distant metastases-free survival, prostate cancer-specific mortality, and OS and reduce the development of castrationresistant prostate cancer. A previous large database study about the effect of DM and metformin in prostate cancer demonstrated that metformin users have reduced recurrence rates when compared with non-metformin users (14). Moreover, patients with DM had a worse OS than those without SM. In a surgical series, metformin was not associated with bRFS in patients who underwent radical prostatectomy (15). Kaushik et al. (16) found that metformin use was not associated with bRFS or OS in their retrospective cohort study (16). Coyle et al. (17) conducted a systematic review and reported that patients receiving prostate cancer radiotherapy had better OS, bRFS, and CSS, which might be related to metformin usage, although no any significant benefit was found for patients who underwent surgery. In the present study, metformin caused a $10 \%$ increase in bRFS rate; however, it was not significant, and results of the present study were similar to those of previous investigations.

In the present study, we also evaluated acute and late side effects and observed that patient with DM were more likely to have acute gastrointestinal and genitourinary side effects. Several previous studies have indicated that DM increases treatmentrelated toxicity in many cancers such as breast, colorectal, and lung cancer $(18,19,20)$. Several theses were put forward about this association. DM might negatively affect leukocyte functions, including chemotaxis, phagocytosis, and insufficient bacterial killing; therefore, it negatively affects host immunity. More tissue damage occurs especially in fast proliferating cells such as the epithelium of the gastrointestinal and genitourinary tract and endothelial tissues after radiotherapy. Consequently, because of endothelial tissue damage, the coagulation system is also activated, resulting in diminished blood flow, thrombosis, and capillary necrosis (21). In patients with DM, endothelial dysfunction is a common reason for morbidity and mortality. Therefore, those with DM would have increased impairment in tissue repair after radiotherapy. 
Gastrointestinal disorders are one of the common complications of DM and include gastroparesis, nonalcoholic fatty liver disease, gastroesophageal reflux disease, and chronic diarrhea (22). Moreover, metformin has some gastrointestinal side effects such as diarrhea. Although patients were asked to report the symptoms that occurred or increased after the start of radiotherapy, it may be sometimes difficult for the patient to tell the difference and distinguish gastrointestinal symptoms related with DM, metformin treatment, or radiotherapy. Some other factors such as androgen deprivation therapy and pelvic field radiotherapy may induce the occurrence and severity of side effects.

\section{Study Limitations}

This study has several limitations. The small sample size, heterogeneous patient characteristics, and retrospective nature of the analysis are the main limitations of this study. Metformin was used in different durations and may influence independently the outcomes of metformin-dependent factors. Moreover, the study did not include a group with DM not treated with metformin. In addition, no analysis was performed on patients with DM who received metformin and did not receive metformin. Furthermore, glycemic control data and hemoglobin A1c levels were not available in this study, which might have some effects on toxicity. Finally, the study had a relatively short follow-up time for observing late side effects and recurrence.

\section{Conclusion}

In this retrospective study, patients with DM and prostate cancer who used metformin and underwent radical radiotherapy have a better bRFS, but significance was not reached. Patients with DM experienced significantly more grade 1-2 acute side effects, whereas a trend toward increased low grades of late side effects was found. Vascular damage in DM may cause impairment in tissue repair after radiotherapy and increase radiotherapyrelated toxicities. Controlled trials in patients with both DM and prostate cancer should be performed to evaluate the effect of DM and metformin usage on outcomes of radiotherapy.

\section{Acknowledgements}

Publication: The results of the study were not published in full or in part in form of abstracts.

Contribution: There is not any contributors who may not be listed as authors.

Conflict of Interest: No conflict of interest was declared by the authors.

Financial Disclosure: The authors declared that this study received no financial support.

\section{Ethics}

Ethics Committee Approval: The retrospective study protocol was approved by the Ethics Committee of Istanbul UniversityCerrahpasa, Cerrahpasa Faculty of Medicine and the study was conducted in accordance with the tenets of the Helsinki Declaration (approved no: 150113 , date: 02.10 .2019 ).
Informed Consent: Retrospective study.

Peer-review: Externally peer-reviewed.

\section{Authorship Contributions}

Supervision: S.Ç.K., Concept: M.D., H.F.Ö.D., Design: H.F.̈.D., Data Collection or Processing: C.B., Analysis or Interpretation: C.Y., Literature Search: C.Y., Writing: M.D.

\section{References}

1. Siegel RL, Miller KD, Jemal A. Cancer statistics, 2018. CA Cancer J Clin 2018;68:7-30.

2. Bullard KM, Cowie CC, Lessem SE, et al. Prevalence of diagnosed diabetes in adults by diabetes type - United States, 2016. MMWR Morb Mortal Wkly Rep 2018;67:359-361.

3. Jameson JL, Fauci AS, Longo D, et al. Harrison's principles of internal medicine. New York: McGraw-Hill CO; 2012. p. 312.

4. Ben Sahra I, Laurent K, Loubat A, et al. The antidiabetic drug metformin exerts an antitumoral effect in vitro and in vivo through a decrease of cyclin D1 level. Oncogene 2008;27:3576-3586.

5. Hamilton RJ, Goldberg KC, Platz EA, et al. The influence of statin medications on prostate-specific antigen levels. J Natl Cancer Inst 2008; 100:1511-1518.

6. Deng D, Yang $Y$, Tang $X$, et al. Association between metformin therapy and incidence, recurrence and mortality of prostate cancer: evidence from a meta-analysis. Diabetes Metab Res Rev 2015;31:595-602.

7. Barone BB, Yeh HC, Snyder CF, et al. Long-term all-cause mortality in cancer patients with preexisting diabetes mellitus: a systematic review and meta-analysis. JAMA 2008;300:2754-2764.

8. Spratt DE, Zhang C, Zumsteg ZS, et al. Metformin and prostate cancer: reduced development of castration-resistant disease and prostate cancer mortality. Eur Urol 2013;63:709-716.

9. Alashkham A, Paterson C, Hubbard S, Nabi G. What is the impact of diabetes mellitus on radiation induced acute proctitis after radical radiotherapy for adenocarcinoma prostate? A prospective longitudinal study. Clin Transl Radiat Oncol 2017;14:59-63.

10. Mohler J, Babaian RJ, Bahnson RR, et al; National Comprehensive Cancer Nerwork. Prostate cancer. Clinical practice guidelines in oncology. J Natl Compr Canc Netw 2007; 5:650-683.

11. Akin $M$, Öksüz DC, Iktueren $B$, et al. Does rectum and bladder dose vary during the course of image-guided radiotherapy in the postprostatectomy setting? Tumori 2014;100:529-535.

12. Nathan DM, Buse JB, Davidson MB, et al; American Diabetes Association; European Association for Study of Diabetes. Medical management of hyperglycemia in type 2 diabetes: a consensus algorithm for the initiation and adjustment of therapy: a consensus statement of the American Diabetes Association and the European Association for the Study of Diabetes. Diabetes Care 2009;32:193-120.

13. Vazquez-Martin A, Oliveras-Ferraros C, Menendez JA. The antidiabetic drug metformin suppresses HER2 (erbB-2) oncoprotein overexpression via inhibition of the mTOR effector p70S6K1 in human breast carcinoma cells. Cell Cycle 2009;8:88-96.

14. Taussky D, Preisser F, Karakiewicz Pl, et al. Impact of diabetes and metformin use on prostate cancer outcome of patients treated with radiation therapy: results from a large institutional database. Can I Urol 2018;25:9509-9515.

15. Allott EH, Hursting SD. Obesity and cancer: mechanistic insights from transdisciplinary studies. Endocr Relat Cancer 2015;22:R365-86. doi: 10.1530/ERC-15-0400.

16. Kaushik D, Karnes RJ, Eisenberg MS, et al. Effect of metformin on prostate cancer outcomes after radical prostatectomy. Urol Oncol 2014;32:43.e1-7. doi: 10.1016/j.urolonc.2013.05.005

17. Coyle C, Cafferty FH, Vale C, Langley RE. Metformin as an adjuvant treatment for cancer: a systematic review and meta-analysis. Ann Oncol 2016;27:2184-2195. 
18. Peairs KS, Barone BB, Snyder CF, et al. Diabetes mellitus and breast cancer outcomes: a systematic review and meta-analysis. J Clin Oncol 2011;29:40-46.

19. Stein KB, Snyder CF, Barone BB, et al. Colorectal cancer outcomes, recurrence, and complications in persons with and without diabetes mellitus: a systematic review and meta-analysis. Dig Dis Sci 2010;55:1839-1851.

20. Zhang XJ, Sun JG, Sun J, et al. Prediction of radiation pneumonitis in lung cancer patients: a systematic review. J Cancer Res Clin Oncol. 2012;138:2103-2116.
21. Turina M, Fry DE, Polk HC Jr. Acute hyperglycemia and the innate immune system: clinical, cellular, and molecular aspects. Crit Care Med 2005;33:1624-1633.

22. Careyva B, Stello B. Diabetes mellitus: management of gastrointestinal Complications. Am Fam Physician 2016;94:980-986. 